\title{
Online Copper Monitoring for Pharmaceutical Processes
}

\author{
Jiyoung Jungr ${ }^{1}$, Junyong $\mathrm{JO}^{2}$ \\ ${ }^{1}$ Penn State University, 120 Ridgevview Dr. Dunmore PA, 18512 USA, \\ ${ }^{2}$ Merck Research Laboratories, 126 E. Lincoln Ave. Rahway NJ 07065, USA \\ Juj23@psu.edu
}

\begin{abstract}
A copper-mediated cyclization which converts ortho-azo anilines to benzotriazoles was utilized to detect residual copper(II) ion in pharmaceutical compounds such as active pharmaceutical ingredient (API) and process intermediates. Adjusting electronic property by both the number of electron donating group (EDG) and the position of EDG remarkably promoted the reactivity toward copper (40times faster) than the benchmark compound. The sensitivity was also markedly increased so the limit of detection is 20 times lower than the benchmark compound. Especially the rapid responses in both color and fluorescence enable the copper probe to be applied as a real-time monitoring tool which was incorporated within high performance liquid chromatography (HPLC). Detail of comparative kinetic studies, structure-reactivity relationships, and its application toward industrial processes will be discussed in the presentation.
\end{abstract}

Key words: Copper probe, Fluorescence, Sensor, Pharmaceutical ingredients, Real-time monitor

\section{Backgrounds}

Copper is the second most used catalyst in pharmaceutical industry [1]. Due to its toxicity health regulatory agencies such as FDA strictly regulated the residual amount of copper in the final drug products [2]. Oxidative cyclization of ortho-azo anilines has been used to detect low level of copper (II) ion [3, 4]. We prepared a series of copper probe molecules which were systematically varied with electron donation groups. Herein we report the fastest responding copper probe and its application to "online" copper monitoring.

\section{Synthesis and Photophysical properties}

Modular synthesis of azo-coupling reaction was used to prepare a series of copper probes. Under mild conditions $\left(2-8^{\circ} \mathrm{C}\right.$ in aqueous solution) generally high isolation yield $(>80 \%)$ was achieved. By systematically change the azo-coupling partners, both the number of electron-donating groups and the position of them were adjusted (Fig. 1). As anticipated, probe 1 exhibits dramatic photophysical property changes upon exposure to copper ion in both absorption and emission (Fig. 2). By cyclization (losing azo-functionality) the original intense red color $\left(\lambda_{\mathrm{abs}}=550 \mathrm{~nm}\right)$ disappears and concomitantly appears yellow absorption band at $400 \mathrm{~nm}$. Formation of benzotriazole elicits strong emission band at $\lambda_{\mathrm{em}}=420 \mathrm{~nm}$ in acetonitrile solution.

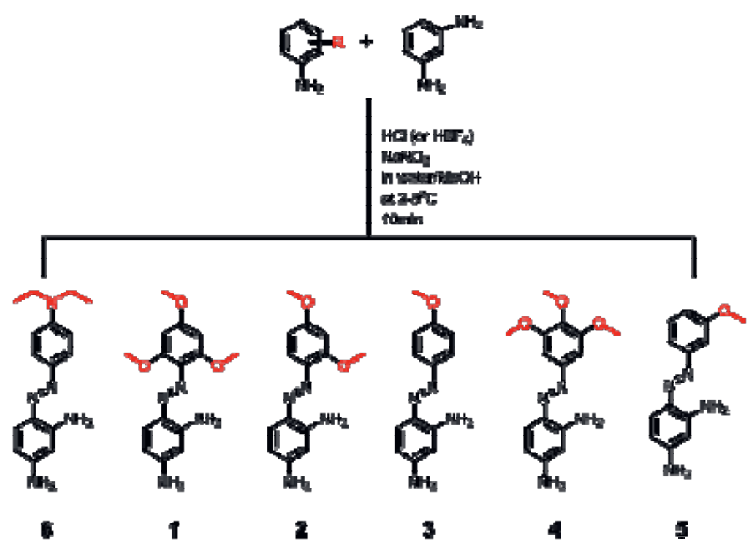

Fig. 1. Synthesis of copper probes 1 through 6.

\section{Comparative Kinetics and Computation}

Systematic changes of electron donating group (EDG) enabled to compare kinetic rates and theoretical reactivity within the series of probes. Having three electron donating groups on orthoand para-position against azo functional group largely promoted the reactivity toward copper ion. 
(a)

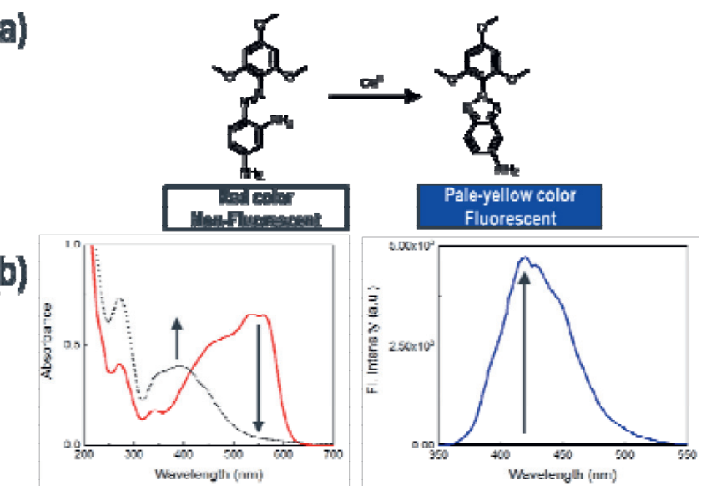

Fig. 2. (a) Chemical structure of probe 1 and (b) UV-vis and Fluorescence spectra prior to and after addition of copper (arrow indicates the changes after the addition of copper)

Similarly theoretical calculation was performed to assess the relative reactivity and the global nucleophilicity index $\left(\omega^{-}\right)$indicate the same trend to the experimental kinetic rates (Tab. 1).

Tab. 1: Rate constants and Global nucleophilicity index $\left(\omega^{-}\right)$

\begin{tabular}{|c|c|c|}
\hline Compound & $k\left(\mathrm{M}^{-1} \mathrm{~s}^{-1}\right)$ & $\omega^{-}$ \\
\hline $\mathbf{1}$ & 355 & 1.173 \\
\hline
\end{tabular}

\begin{tabular}{|l|l|l|}
\hline $\mathbf{2}$ & 5.0 & 1.146 \\
\hline $\mathbf{6}$ & 9.4 & 1.124 \\
\hline
\end{tabular}

\section{Proof-of-principle: Real-time monitoring of copper}

Rapid response toward copper enabled to apply the probe into real-time detection of copper in a reaction stream. As a proof-of-principle, high performance liquid chromatography (HPLC) system was utilized (Fig 2a). Quadruple pump in HPLC system delivers precise amount of each mobile phase where mobile phase bottles were filled with copper probe $(80 \mu \mathrm{M})$, copper standard solution ( $250 \mathrm{mM}$ in water) and water, respectively. The mobile phase mixer is mixing each mobile phase as programed to adjust copper concentration. The internal system volume of HPLC system provides the reaction vessel and UV detector was used to monitor the absorbance at $210 \mathrm{~nm}$ and 550nm (Fig $2 \mathrm{~b}$ ). As continuously changing copper concentration, the corresponding signal at $210 \mathrm{~nm}$ shows the same up-down with $30 \mathrm{sec}$ delay (presumably from the system volume). Exactly opposite signal was observed at $550 \mathrm{~nm}$ where the maximum absorbance of azo-compound.

(a)
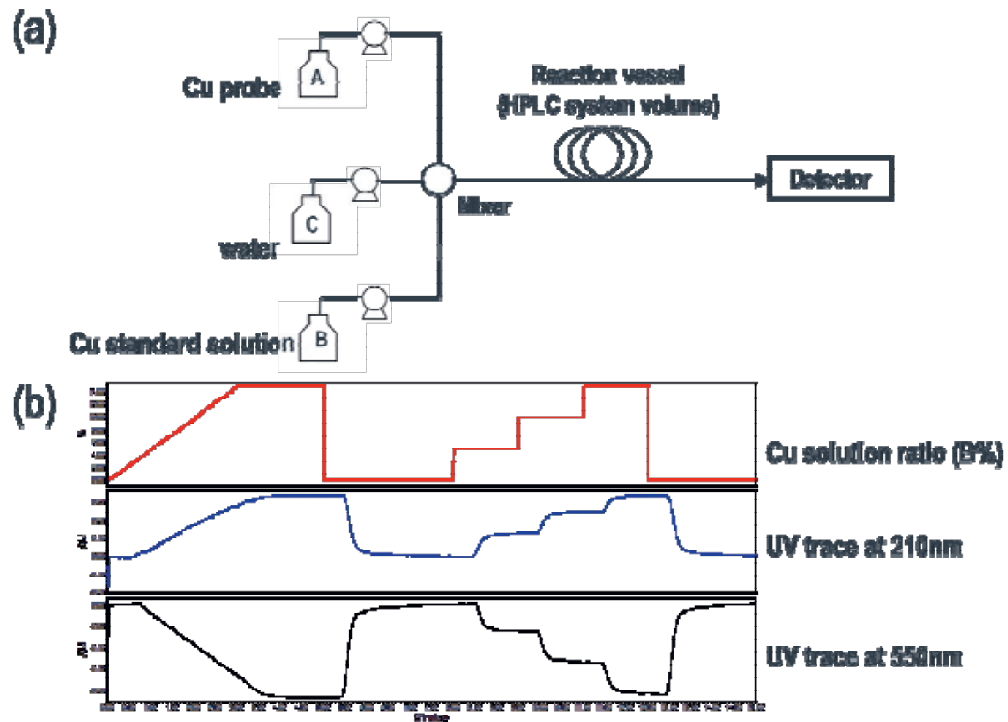

Fig.2. (a) schematic diagram of HPLC setup for real-time monitoring of copper and (b) plot between copper concentrations vs UV signals.

\section{References}

[1] M. L. Crawley, B. M. Trost, Applications of Transition Metal Catalysis in Drug Discovery and Development: An Industrial Perspective, 2012, doi: $10.1002 / 9781118309872$

[2] ICH Guideline for Elemental Impurities. http://www.ich.org/products/quidelines/quality/qua lity-single/article/guideline-for-elementalimpurities.html

[3] J. Jung, J. Jo, A. Dinescu, Org. Process Res. Dev. 21, 1689-1693 (2017); doi: 10.1021/acs.oprd.7b00269

[4] J. Jo, H.Y, Lee, W. Liu, A. Olasz, C-H. Chen, D. Lee, J. Am. Chem. Soc. 134, 16000-16007 (2012); doi: 10.1021/ja307316s 